\title{
Innate immunity: quo vadis?
}

\author{
Ruslan Medzhitov \\ The next decade will probably witness the development of new concepts that will incorporate the presently \\ unexplained aspects of innate immunity.
}

\begin{abstract}
"If you don't know where you are going, you will wind up somewhere else."
\end{abstract}

Yogi Berra

\begin{abstract}
The field of innate immunity has enjoyed tremendous progress in the past 10 years. This is due in large part to identification of the pathways and mechanisms of innate immune recognition and innate control of adaptive immunity. In the next decade, many developments can be expected that will better define already known aspects of the innate immune system, including the characterization of additional pattern-recognition receptors (PRRs), their signaling pathways and their roles in host defense. Other developments may not be as obvious or may be entirely unexpected. This commentary will discuss a few of the possible future developments of the second type.
\end{abstract}

\section{Microbial sensors: PRRs and beyond}

Several families of PRRs have been characterized so far, including Toll-like receptors (TLRs), intracellular Nod proteins, Nodlike receptors, RIG-like receptors, dectin proteins and several others ${ }^{1}$. These receptor families have now been well established to have important roles in pathogen recognition and the activation of different arms of innate and adaptive immunity. Several pathways of pathogen recognition are still not accounted for, including one or more additional cytosolic DNA sensors and cellintrinsic sensors that detect retroviruses. It can also be expected that innate immunerecognition pathways that are not based on pattern recognition will also be identified over the next decade.

Howard Hughes Medical Institute, Department of Immunobiology, Yale University School of Medicine, New Haven, Connecticut, USA.

e-mail: ruslan.medzhitov@yale.edu
One important area of future investigation is whether the innate immune system can detect pathogen-specific features (biochemical activities or particular modes of interactions with the host cells) so that it could distinguish between pathogenic and commensal microorganisms. Such a recognition system has been proposed (as a 'guard theory') to operate in plants, in which dedicated products of resistance genes are thought to monitor key cellular processes that are common targets for pathogens, for example, endosomal trafficking and cytoskeletal dynamics ${ }^{2}$. Alterations in normal cellular processes are thought to be sensed by such gene products (referred to as 'guard proteins') to initiate host-defense responses in plants. Similar microbe-sensing strategies may operate in mammalian cells. Indeed, the NLRP3 inflammasome seems to sense membrane integrity, as it can be activated by diverse stimuli that can disrupt cellular membranes. Both pore-forming toxins of Gram-positive pathogens and type III secretion systems of Gram-negative pathogens can be detected by NLRP3 inflammasomes, which results in the secretion of members of the interleukin 1 family and many other leaderless proteins by the caspase-1-dependent nonconventional secretory pathway ${ }^{3}$. Additional mechanisms that can sense pathogen-specific effects on the host probably exist, and they may complement pattern recognition and missing-self recognition.

Whether the detection of pathogenspecific activities is essential for pathogencommensal discrimination remains to be established, however. The argument here can go both ways: on the one hand, the mammalian immune system is widely assumed to be able to distinguish pathogens from commensals to mount immune responses to the former and to avoid responding to the latter; however, commensals do not seem to be intrinsically different from pathogens in terms of their ability to induce an immune response. In fact, commensal-specific innate and adaptive immune responses occur normally and seem to be needed to maintain the normal host-commensal homeostasis ${ }^{4}$. Thus, the immune system provides protection not only from pathogens but also from commensals. Furthermore, the distinction between commensals and pathogens is operational (that is, whether or not they can cause a disease) and is conditional on the host identity and its immune status. Both commensal and pathogenic microbes use the host as a niche and use diverse adaptation strategies to establish successful colonization. The full diversity of adaptation strategies used by commensal microbes is unknown at present but it is probably at least as great as the diversity of strategies used by pathogens. The relationship of these strategies to the host's sensing abilities will need to be systematically analyzed in future studies to clarify whether or not the immune system is able to detect some adaptation strategies (for example, virulence activities) but not others. Ultimately, a more biological classification of the microbes that colonize the host may be based on their hostadaptation strategies rather than on their ability to cause a disease. Indeed, virulence factors are nothing other than gene products that evolved for adaptation to a particular niche in the host. Accordingly, the additional pathways of innate immune sensing may best be identified on the basis of common themes in microbial adaptation to the host.

\section{Effects of microbiota on the host}

Commensal microflora has long been recognized to have a variety of beneficial effects on host development and physiology. The composition of the microbial communi- 


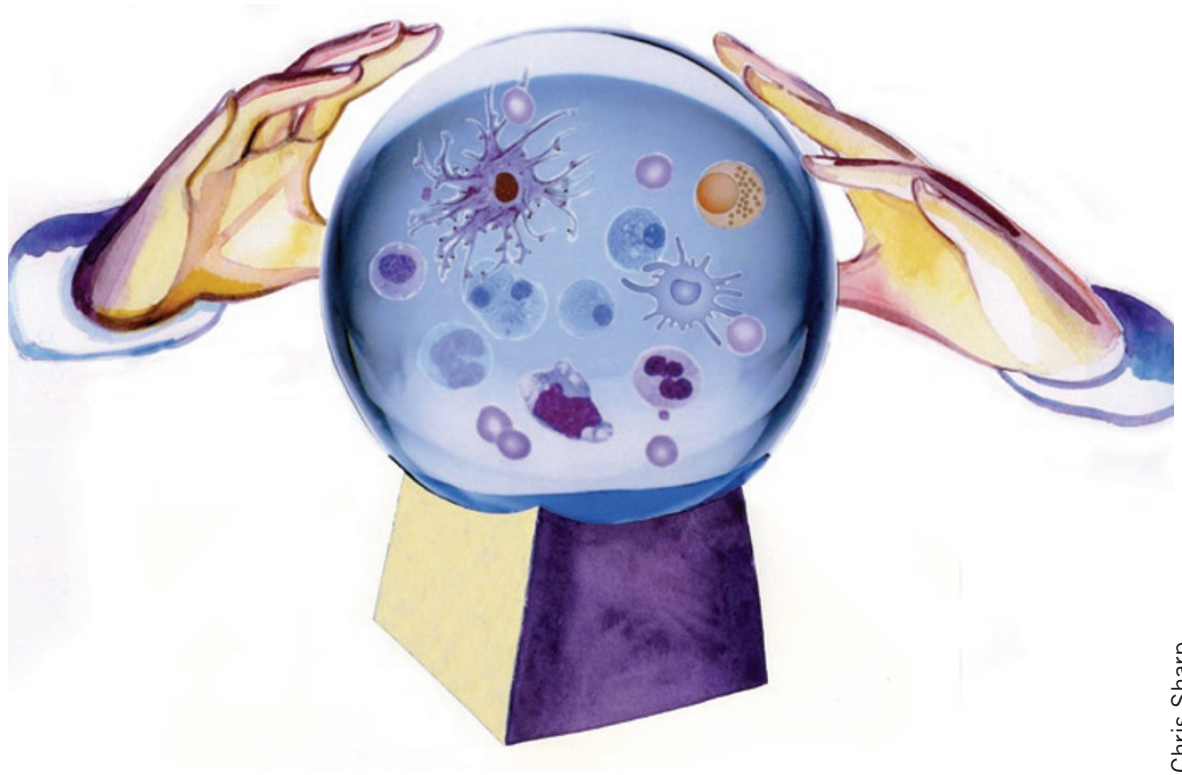

What does the future hold for innate immunity research?

ties that colonize the host, particularly in the lower gastrointestinal tract, is difficult to evaluate because many commensal bacteria are unculturable obligatory anaerobes. However, studies have begun to demonstrate remarkably specific and profound effects of seemingly subtle variations in microbial composition on host immunity and susceptibility to diseases ${ }^{5,6}$. Many discrepant results obtained in the past in different laboratories can presumably be ascribed to the differences in the composition of commensal communities in different animal facilities throughout the world. Whether just a few bacterial or viral species make all the difference is not yet clear, and it is possible that more complex microbial communities are responsible for the variations in the effects of commensal flora on the host. A major challenge for future studies is to identify the full spectrum and mechanisms of the effects of microbiota on the immune system, on susceptibility to infection and autoimmune disease, and on other physiological and pathological processes.

\section{Recognition of parasites and allergens}

One area of investigation in innate immune recognition that has not yet seen much progress is study of the recognition of multicellular parasites. No receptors have yet been clearly demonstrated to be involved in sensing parasite infections, and consequently, the innate control mechanisms of $\mathrm{T}$ helper type $2\left(\mathrm{~T}_{\mathrm{H}} 2\right)$ immune responses are not well defined. Indeed, in the field of type $2 \mathrm{immu}-$ nity, there is no consensus even in terms of very basic questions about the mechanisms involved in the induction of $\mathrm{T}_{\mathrm{H}} 2$ responses. There is also a major gap in knowledge relating to the mechanisms of innate immune recognition of allergens. Allergens are noninfectious environmental antigens that have immunogenic activities that can trigger $\mathrm{T}_{\mathrm{H}}{ }^{2-}$ immunoglobulin $\mathrm{E}$ immune responses. Why allergens are immunogenic is, in general, not well understood, and the mechanisms of their immunogenicity are largely obscure. Notably, there are several different biochemical classes of allergens, and each probably activates immune responses through distinct mechanisms. Some allergens seem to mimic the biochemical activities of parasiteassociated enzymes, for example, proteases. These enzymatic activities may be detected by dedicated sensors of parasitic infection ${ }^{7}$. This form of innate immune recognition is thus based on the sensing of unique activities of parasites rather than direct molecular (pattern) recognition. Other allergens, specifically those that have lipid-binding properties, are immunogenic because of physical association with lipopolysaccharide and perhaps other TLR ligands ${ }^{8}$. However, in most cases, the mechanisms of innate immune recognition of allergens, as well as of parasitic worms, are unknown, and this remains a major deficiency in the understanding of innate immune recognition.

\section{Immunity versus immunogenicity}

It is now well appreciated that innate immune recognition, particularly by PRRs, is the basis of immunogenicity of microbial stimuli, such as lipopolysaccharide and nucleic acids. It is also well established that activation of PRRs results in the induction of adaptive immune responses. Although this has been most clearly demonstrated for TLRs, other receptor families are also coupled to the induction of adaptive immunity. Thus, the rules of immunogenicity are more or less understood, but what is not known are the rules for the induction of protective immunity. Indeed, not all immune responses are protective against a given pathogen. It is known that induction of the appropriate effector class is required, but beyond that, the parameters of protective immunity are not fully understood. For example, not all antigen specificities can confer pathogen clearance. Because host protection from a pathogen is what ultimately matters, it is possible that specific mechanisms exist that instruct immune responses in a way that maximizes the chances of protection. These mechanisms probably operate at the level of the innate immune system, similar to the innate control mechanisms that determine the choice of effector responses of T cells and B cells. Lack of understanding of the rules of induction of protective immunity explains why the rational design of effective vaccines remains unknown. Such knowledge would clearly be very critical for future efforts in vaccine development.

\section{Resistance and tolerance to infections}

It has been recognized for decades in the field of plant immunity that there are two fundamentally different strategies of host defense from infection: resistance and tolerance ${ }^{9,10}$. Mechanisms of resistance diminish pathogen burden through pathogen detection and elimination. Mechanisms of tolerance, however, diminish the negative effects on host fitness of a given amount of pathogen burden. Although the mechanisms of resistance have been the main subject of immunology, with a few notable exceptions ${ }^{11-14}$, mechanisms of tolerance to infection have not been studied in animals. A few available studies ${ }^{11-14}$ suggest that tolerance has a very important role in host-pathogen interactions, and future research will need to focus on systematic analysis of the mechanisms that allow the host to endure a given amount of pathogen assault. Manipulation of the host tolerance mechanisms may be a valuable therapeutic strategy for some infectious diseases, as exemplified by a study of tolerance to Plasmodium chabaudi chabaudi infection $^{14}$.

Infection can negatively affect host fitness through two distinct mechanisms. First, pathogens can directly cause tissue damage through their virulence activities. Second, 
tissue damage can be caused by the immune and inflammatory responses to the pathogen. Thus, host tolerance to infection must come in two forms to enable tolerance to the two sources of tissue damage. The cellular and molecular mechanisms responsible are largely unknown, and these represent important subjects for future research.

'Nonimmune' functions of innate immunity The dominant role of the immune system is to protect the host from infectious agents. However, the immune system may have other roles in mammalian biology. Thus, TLRs have been demonstrated to be involved in tissue repair and homeostasis, at least in tissues colonized by microbes, such as the colon. Available evidence suggests that TLRs and inflammasomes may also have a role in sterile inflammation and have important roles in the pathogenesis of inflammatory diseases ${ }^{3,15}$. In addition, the innate immune system may have a role in the defense against noninfectious noxious insults, such as toxic xenobiotics, environmental irritants and venoms. Characterization of the putative functions of the innate immune system in these contexts is an exciting area for future research.

\section{Conclusions}

Despite the tremendous progress made by studies of the innate immune system, many fundamental questions remain and some new questions will surely arise in the near future. The pattern-recognition theory proposed by Janeway over 20 years ago ${ }^{16}$ has served as a conceptual background for understanding the innate immune system. Additional concepts must now be developed to explain more enigmatic aspects of host-microbe interactions and the functions of the innate immune system in broader physiological contexts. As Yogi Berra pointed out, "It is hard to make predictions, especially about the future," but it is safe to say that exciting new discoveries in the field of innate immunity will continue in the next decade and beyond.

\section{COMPETING FINANCIAL INTERESTS}

The author declares no competing financial interests.

1. Takeuchi, O. \& Akira, S. Cell 140, 805-820 (2010).

2. Dangl, J.L. \& Jones, J.D. Nature 411, 826-833 (2001).

3. Martinon, F., Mayor, A. \& Tschopp, J. Annu. Rev. Immunol. 27, 229-265 (2009).

4. Shiloh, M.U. et al. Immunity 10, 29-38 (1999).

5. Gaboriau-Routhiau, V. et al. Immunity 31, 677-689 (2009).

6. Ivanov, I.I. et al. Cell 139, 485-498 (2009).

7. Sokol, C.L., Barton, G.M., Farr, A.G. \& Medzhitov, R Nat. Immunol. 9, 310-318 (2008).

8. Trompette, A. et al. Nature 457, 585-588 (2009).

9. Read, A.F., Graham, A.L. \& Raberg, L. PLoS Biol. 6, e4 (2008).

10. Schneider, D.S. \& Ayres, J.S. Nat. Rev. Immunol. 8 889-895 (2008).

11. Ayres, J.S., Freitag, N. \& Schneider, D.S. Genetics 178, 1807-1815 (2008).

12. Ayres, J.S. \& Schneider, D.S. PLoS Biol. 7, e1000150 (2009).

13. Raberg, L., Sim, D. \& Read, A.F. Science 318, 812 814 (2007).

14. Seixas, E. et al. Proc. Natl. Acad. Sci. USA 106 15837-15842 (2009).

15. Rock, K.L. \& Kono, H. Annu. Rev. Pathol. 3, 99-126 (2008).

16. Janeway, C.A. Jr. Cold Spring Harb. Symp. Quant. Biol. 54, 1-13 (1989). 